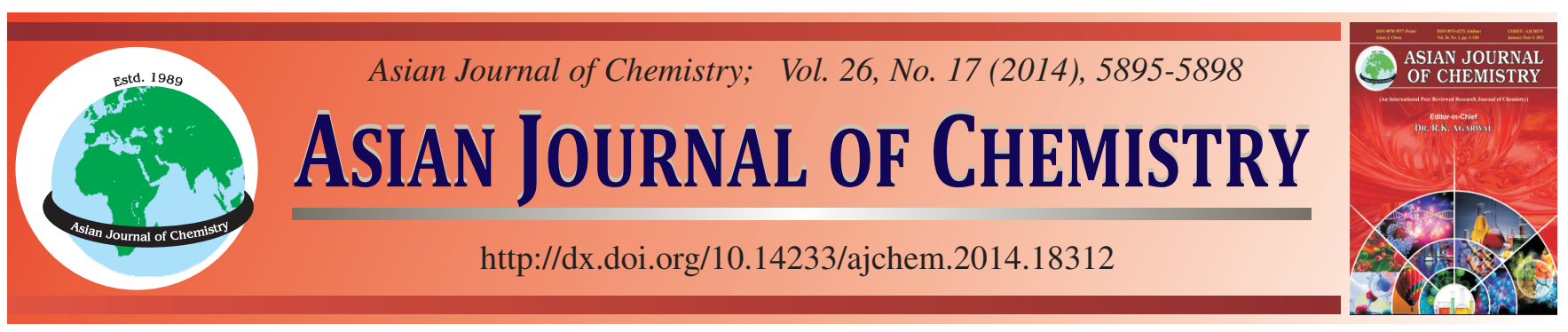

\title{
Preparation and Properties of Chitosan/Nanocrystalline Cellulose Composite Films for Food Packaging $\dagger$
}

\author{
Feng Dong ${ }^{1,2}$, Shujun Li ${ }^{1, *}$, Meiling Yan $^{1}$ and Chunje Li $^{1}$
}

\begin{abstract}
${ }^{1}$ Key Laboratory of Bio-based Material Science and Technology of Ministry of Education, Northeast Forestry University, Harbin, P.R. China
\end{abstract} ${ }^{2}$ Light Industry and Textile College, Qiqihar University, Qiqihar, P.R. China

*Corresponding author: E-mail: 84881619@qq.com

Nanocrystalline cellulose was prepared from microcrystalline cellulose using the ultrasonication. Nanocrystalline cellulose reinforced chitosan films were prepared by solution casting. The incorporation of nanocrystalline cellulose significantly enhanced the mechanical properties. The dry and wet tensile strength of nanocomposite films were improved by 22.4 and $542 \%$, respectively, for the nanocomposite containing $30 \mathrm{wt} \%$ of nanocrystalline cellulose. The swelling behaviour was studied by analyzing the water uptake of the nanocomposite films. SEM of the nanocomposite films was found that $30 \mathrm{wt} \%$ of nanocrystalline cellulose was dispersed homogenously into chitosan matrix. Nanocrystalline cellulose played an important role in improving the mechanical and barrier properties of chitosan film.

Keywords: Chitosan, Nanocrystalline cellulose, Nanocomposite films, Mechanical property, Swelling property.

- - - - - - - - - - - - - - - - - - - - - - - - - - -

\section{INTRODUCTION}

Chitosan and cellulose are an important classes of biological polymers. They have been of increasing interest as polymeric renewable material in the last few decades, not only because they are naturally abundant, eco-friendly and sustainable, but also because of their attractive properties. Nanocrystalline cellulose (NCC) were applied as an attractive component for reinforcement of polymers ${ }^{1-7}$, due to its exceptional mechanical properties (high specific strength and modulus), large specific surface area, high aspect ratio, environmental benefits and low $\operatorname{cost}^{8,9}$. Nanocrystalline cellulose can be produced by mechanical processes, such as pulping beating ${ }^{10}$, high pressure homogenizing $^{11}$, cryo-crushing ${ }^{12}$ and ultrasonication ${ }^{13}$.

Chitosan (CS) is non-toxic, biodegradable, biofunctional, biocompatible $^{14}$. Chitosan films can be applied in various fields such as waste treatment, packaging, food processing and medicine ${ }^{15-19}$, but the disadvantages of the chitosan films lay in its relatively high water vapor permeability. Chitosan and cellulose have similar chemical structures and are expected to possess excellent biocompatibility and biodegradability when compounded into hybrids. With the aim of developing convenient, efficient, environmentally benign method on the properties of NCC-CS nanocomposites, NCC was prepared from the microcrystalline cellulose (MCC) using ultrasonication without any additional chemical treatments ${ }^{20}$. The reinforcing capability of the obtained NCC was examined by adding it to chitosan. The mechanical properties at dry and wet conditions and the water vapor permeability properties of NCC-CS nanocomposite films were investigated. NCC-CS nanocomposite films are also expected to satisfy the basic requirements for food packaging applications.

\section{EXPERIMENTAL}

Preparation of nanocrystalline cellulose: Together with $100 \mathrm{~mL}$ distilled water, $0.5 \mathrm{~g}$ microcrystalline cellulose (Philadelphia, PA, USA) was mixed into a $500 \mathrm{~mL}$ beaker and swelled for $24 \mathrm{~h}$. The obtained mixture was split into three portions and was then treated using a JY98-IIID ultrasonicator (Ningbo Scientz Biotech Co. Ltd., Ningbo, China) for $0.5 \mathrm{~h}$ at an output power of $1500 \mathrm{~W}$. Nanocrystalline cellulose was successfully prepared. After that, the formed colloid suspension was freeze-dried and stored at $5^{\circ} \mathrm{C}$ for further testing.

Preparation of NCC-CS nanocomposite films: $1 \mathrm{wt} \%$ chitosan (degree of deacetylation: 88-89\%) water dispersion was prepared by dissolving the polysaccharide in $2 \%$ aqueous acetic acid at $40{ }^{\circ} \mathrm{C}$ for $1.5 \mathrm{~h}$ under stirring. Subsequently, the desired amount of NCC suspension $(0.1 \mathrm{wt} \%$ was added into the CS solution. The mixture was then stirred at $1000 \mathrm{rpm}$ for $2 \mathrm{~h}$, followed by sonication for $10 \mathrm{~min}$ to remove the bubbles. 
After that, the NCC-CS suspension was poured onto the glass pane and air-dried for $24 \mathrm{~h}$, at room temperature at $35 \%$ relative humidity $(\mathrm{RH})$ to allow water to evaporate to form a film. The thicknesses of final films were between 15 and 20 $\mu \mathrm{m}$. The nanocomposite films containing $0,10,20$ and $30 \mathrm{~g}$ suspension of NCC were prepared and were designated as CS, CS10, CS20 and CS30, respectively.

\section{Characterization of NCC and NCC-CS nanocomposites}

Transmission electron microscopy (TEM): Transmission electron microscopy(TEM) observations were performed with an FEI/Philips Tecnai G2(Philips, Eindhoven, The Netherlands) operating at $80 \mathrm{kV}$. A drop of dilute NCC suspension was deposited on carbon-coated grids. The NCC was stained with $2 \%$ phosphotungstic acid. Nanocrystalline cellulose was dried at room temperature for $c a .0 .5 \mathrm{~h}$.

Scanning electron microscopy (SEM): Scanning electron microscopy(SEM) of NCC-CS nanocomposite films were obtained on an InspectF (FEI) operating at $10 \mathrm{kV}$. Prior to SEM observations, samples were sputter-coated with gold to enhance conductivity.

Thermogravimetric analysis (TGA): Thermogravimetric analysis of the samples were carried out using a TGA analyser (STA 449 F3 Jupiter, NETZSCH Co. Ltd., Germany). Experiments were carried out under nitrogen atmosphere. The weight of the samples varied from 4-6 mg, scanning range was maintained to $20-600{ }^{\circ} \mathrm{C}$ and the heating rate was $10^{\circ} \mathrm{C}$ $\min ^{-1}$.

Ultraviolet-visible spectrometry: Ultraviolet-visible spectrometry (UV-VIS, Varian Cary 50 Bio Spectrophotometer) was used to measure the light transmittance of the NCCCS nanocomposite films in the range of 300-500 nm.

Mechanical testing: Tensile strength (TS), tensile modulus (TM) and elongation at break (Eb) of the films were measured by using Universal Testing Machine (AG-1, Shimadzu, Japan) at a speed of $20 \mathrm{~mm} / \mathrm{min}$. The distance between the jaws was set at $50 \mathrm{~mm}$. Five samples were tested with dimensions of $100 \mathrm{~mm}$ in length and $15 \mathrm{~mm}$ in width and the film thickness was measured at five random positions along the film.

Swelling property: The test samples were first dried at $37{ }^{\circ} \mathrm{C}$ for $12 \mathrm{~h}$ in an incubator and then accurately weighed. The dried films were then immersed in distilled water for 20$150 \mathrm{~s}$. The wet weight of the films was measured by taking out the films from the water and blotting with a filter paper to remove the surface adsorbed water followed by immediately weighing the films. The swelling property of the films was calculated by the following equation ${ }^{21}$ :

$$
\mathrm{S}=\left[\frac{\mathrm{W}_{\mathrm{s}}-\mathrm{W}_{\mathrm{d}}}{\mathrm{W}_{\mathrm{d}}}\right] \times 100
$$

where, $\mathrm{S}$ is the percentage of water absorption of the films at equilibrium; Ws and Wd are the weights (g) of the samples in the dry and swollen states, respectively.

For a plane sheet geometry and if the original concentration of water in the solid boundary is zero and the polymer is placed in an infinite bath of water, the solution is given $b y^{22}$ :

$$
M_{(t)}-\frac{M_{0}}{M_{(e q)}}=\frac{4}{L}\left[\frac{D t}{\pi}\right]^{1 / 2}
$$

where $\mathrm{L}$ is the thickness of the films, $\mathrm{D}$ is the diffusion coefficient, $\mathbf{M}_{(\mathrm{t})}$ and $\mathrm{M}_{(\mathrm{eq})}$ are the swelling level of the films at times $\mathrm{t}$ and the equilibrium swelling level, respectively.

\section{RESULTS AND DISCUSSION}

Transmission electron microscopy analysis: Fig. 1 shows that the obtained NCC had regular rod shaped with sizes in the diameter range of 5-10 nm, primarily composed of single cellulose elementary fibril. Nanocrystalline cellulose was successfully prepared with ultrasonic treatments.

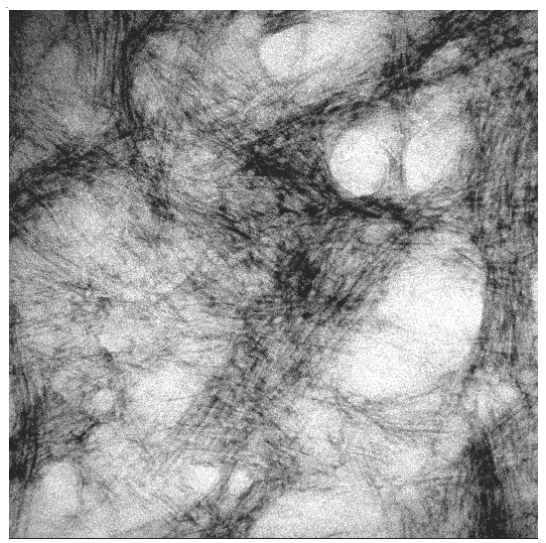

Fig. 1. TEM image of nanocrystalline cellulose

Surface morphology of nanocomposite films analysis: Fig. 2 represents SEM micrographs of the cross-section of nanocomposite films. It is clear that the addition of NCC caused changes in the film microstructure, since the non-reinforced CS film (Fig. 2a) exhibited a smooth surface with few cracks, as required for a homogeneous material. By comparing the micrographs of CS to that of the CS30, nanocomposite films exhibited a homogeneous and dense structure, indicating a proper dispersion of NCC into chitosan matrix. The SEM results support the enhanced mechanical and barrier properties of chitosan films due to the addition of NCC.
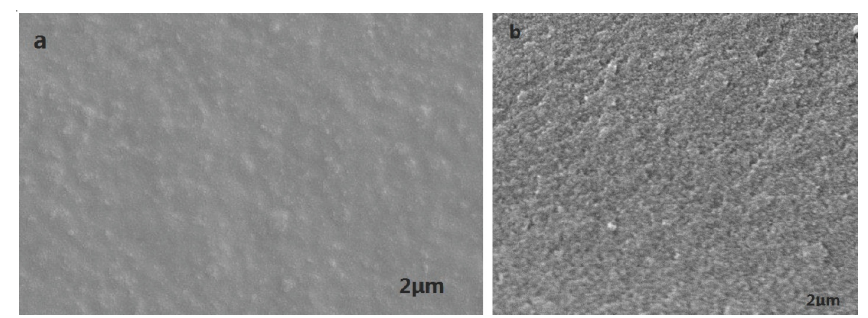

Fig. 2. SEM images of the cross-section of (a) CS and (b) CS30

Thermal property of nanocomposite films: TGA curves were represented in Fig. 3. Nanocrystalline cellulose was stable during the heating range $25-280^{\circ} \mathrm{C}$, losing only $6.8 \%$ of its initial weight. The weight loss for the CS, CS20 and CS30 were $32,39.25$ and $41.9 \%$, respectively. For all the samples, a major weight loss was found at around $280^{\circ} \mathrm{C}$. All chitosan samples displayed a similar thermal behaviour at the temperature range $280-600{ }^{\circ} \mathrm{C}$ and the influence of NCC on the thermal stability was found to be negligible. So, apart from a small increase in the heat flow, the effect of NCC addition in the thermal property of the chitosan films was not significant. 


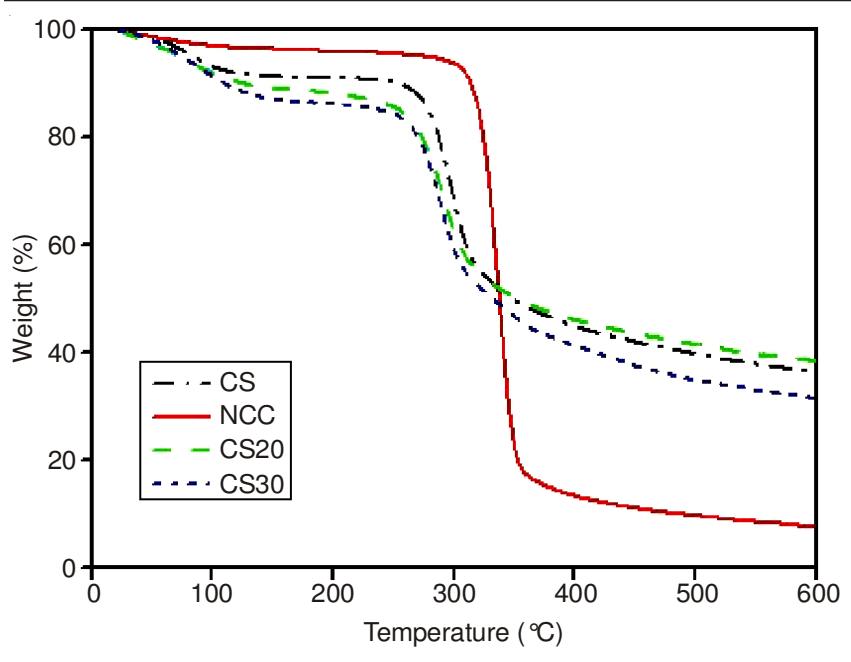

Fig. 3. TGA curves for NCC, CS, CS20 and CS30

UV-visible transmittances analysis: The percent transmittances at 300, 400 and $500 \mathrm{~nm}$ are tabulated in Table-1. The transmittance values of CS studied here are $86.8 \%$ at $400 \mathrm{~nm}$ and $90.7 \%$ at $500 \mathrm{~nm}$. With increasing content of NCC, the percent transmittance decreases slowly compared to CS. As expected, there is some scattering and absorption from the NCC-CS interface in scales from nanometers to micrometers, resulting in a moderate loss transmission for nanocomposites. Nevertheless, the NCC-CS nanocomposite films still shows good transparency when the NCC content is $30 \mathrm{wt} \%$.

\begin{tabular}{cccc}
\multicolumn{5}{c}{ TABLE-1 } \\
\multirow{3}{*}{ UV-VIS TRANSMITTANCES OF NANOCOMPOSITE } \\
FILMS AT 300, 400 AND 500 nm \\
\cline { 2 - 4 } Sample & \multicolumn{3}{c}{ Transmittace (\%) } \\
\cline { 2 - 4 } & $300 \mathrm{~nm}$ & $400 \mathrm{~nm}$ & $500 \mathrm{~nm}$ \\
\hline CS & 64.5 & 86.8 & 90.7 \\
CS10 & 53.6 & 77.9 & 81.4 \\
CS20 & 50.2 & 71.4 & 76.8 \\
CS30 & 44.3 & 65.2 & 71.3 \\
\hline
\end{tabular}

Analysis of mechanical properties: The mechanical properties are summarized in Table-2. For dry samples, it can be seen that tensile strength increases with increasing NCC content. The tensile strength of CS20 and CS30 are 11.4 and $23.4 \%$ higher than that of CS, respectively. Young's modulus for CS30 is improved by $83.3 \%$ in comparison with CS. For wet samples, the tensile strength of CS immersed in distilled water for $180 \mathrm{~s}$ is $1.9 \mathrm{MPa}$, which is much lower than the value for dry CS. The tensile strength of NCC-CS nanocomposites immersed in distilled water for $180 \mathrm{~s}$ increases with increasing NCC content. The wet tensile strength is improved by 4.68 and $5.42 \%$ for CS20 and CS30 respectively, compared to the value for wet $\mathrm{CS}$. In contrast, the elongation at break for the wet samples decreases with increasing NCC content, which is of the same trend as in dry conditions. The reason may results from the NCC particles and the CS transfer to the nanocrystal network, leading to more uniform stress distribution and minimization of the stress concentration area. Thus, NCC particles act as a good reinforcing agent in chitosan films ${ }^{23,24}$.

Analysis of swelling property: Experimental data for the swelling level within distilled water for nanocomposite films are shown in Fig. 4. It clearly shows that all nanocomposite samples absorb water rapidly within the first $20 \mathrm{~s}$ and gradually reach the equilibrium within $150 \mathrm{~s}$. The equilibrium swelling degree decreases from $1100 \%$ for CS to $220 \%$ for CS30 with increasing content of NCC. The incorporation of NCC significantly reduces the equilibrium swelling level of the CS matrix. The diffusion of water into NCC-CS nanocomposite film follows Fick's law in the whole swelling process. The value of D estimated by Fick's law for the CS film and CS30 here are $1.72 \times 10^{-9}$ and $1.27 \times 10^{-9} \mathrm{~cm}^{2} \mathrm{~s}^{-1}$, respectively.

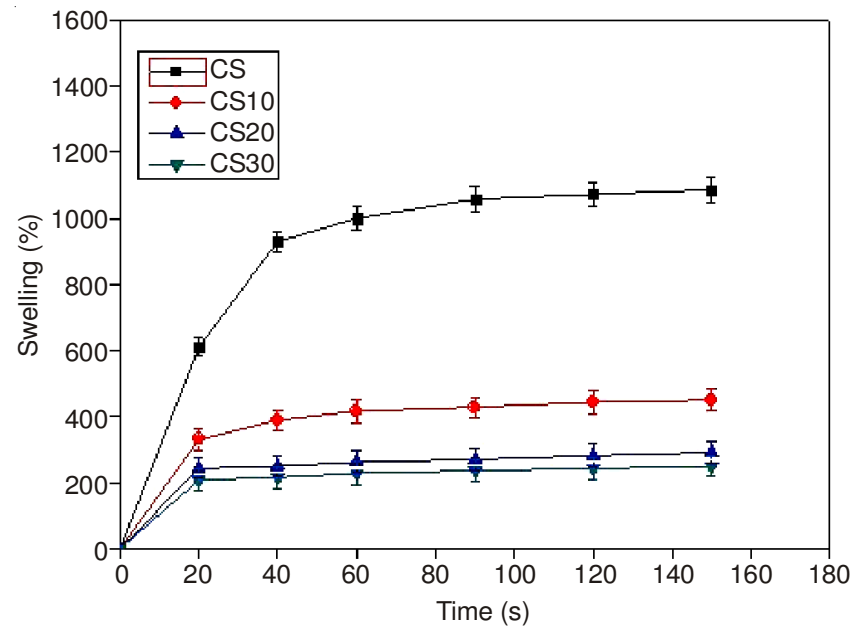

Fig. 4. Swelling level for nanocomposite films

\section{Conclusion}

It was observed that NCC acted as a good reinforcing agent in chitosan and $30 \%$ of NCC loading gave the best mechanical property and swelling property. Under the dry conditions, the tensile strength and Young's modulus of chitosan increased with increasing NCC content because of the reinforcement of $\mathrm{NCC}$, while the elongation at break decreased. The improvements of tensile strength and Young's modulus in wet samples were larger than those for dry samples. Nanocrystalline cellulose also improved the barrier properties of the chitosan by reducing swelling property. Surface morphology of the nanocomposite films revealed a homogeneous

TABLE-2

MECHANICAL PROPERTIES OF NANOCOMPOSITE FILMS

\begin{tabular}{|c|c|c|c|c|c|c|}
\hline \multirow{2}{*}{ Sample } & \multicolumn{2}{|c|}{ Tensile strength (MPa) } & \multicolumn{2}{|c|}{ Elongation at break (\%) } & \multicolumn{2}{|c|}{ Young'smodulus (MPa) } \\
\hline & Dry & Wet & Dry & Wet & Dry & Wet \\
\hline CS & $47.5 \pm 12.9$ & $1.9 \pm 0.3$ & $37.5 \pm 6.6$ & $85.4 \pm 10.2$ & $1800 \pm 300$ & $0.9 \pm 0.1$ \\
\hline CS10 & $50.9 \pm 15.2$ & $4.7 \pm 1.8$ & $19.5 \pm 6.8$ & $38.7 \pm 7.4$ & $2200 \pm 700$ & $7.3 \pm 1.4$ \\
\hline CS20 & $52.9 \pm 13.8$ & $10.8 \pm 3.4$ & $15.7 \pm 7.2$ & $33.6 \pm 8.2$ & $2400 \pm 500$ & $18.9 \pm 4.1$ \\
\hline CS30 & $58.6 \pm 16.2$ & $12.2 \pm 2.2$ & $17.6 \pm 3.5$ & $23.4 \pm 5.7$ & $3300 \pm 600$ & $25.8 \pm 7.3$ \\
\hline
\end{tabular}

Wet samples were obtained by immersion in water for 150 s to reach equilibrium. 
structure was indicating proper dispersion of the NCC into the chitosan matrix. Overall, NCC-CS nanocomposite films due to their excellent mechanical and swelling properties should have a promising impact in food packaging over the coming years.

\section{ACKNOWLEDGEMENTS}

This work was supported by the Fundamental Research Funds for the Central Universities (DL11EB01).

\section{REFERENCES}

1. M.A.S. Azizi Samir, F. Alloin, J.-Y. Sanchez and A. Dufresne, Polymer, 45, 4149 (2004)

2. N. Ljungberg, J.-Y. Cavaillé and L. Heux, Polymer, 47, 6285 (2006).

3. N.L. Garcia de Rodriguez, W. Thielemans and A. Dufresne, Cellulose, 13, 261 (2006).

4. X.D. Cao, H. Dong and C.M. Li, Biomacromolecules, 8, 899 (2007).

5. A.N. Nakagaito and H. Yano, Appl. Phys., A Mater. Sci. Process., 78, 547 (2004)

6. H.M. Azeredo, L.H. Mattoso, R.J.A. Avena-Bustillos, G.C. Filho, M.L. Munford, D. Wood and T.H. McHugh, J. Food Sci., 75, N1 (2010).

7. X. Cao, Y. Chen, P.R. Chang, A.D. Muir and G. Falk, Polymer Lett., 2, 502 (2008).
8. Y. Habibi, L.A. Lucia and O.J. Rojas, Chem. Rev., 110, 3479 (2010).

9. M.M. de Souza Lima and R. Borsali, Rapid Commun., 25, 771 (2004).

10. A.N. Nakagaito and H. Yano, Appl. Phys., A Mater. Sci. Process., 78, 547 (2004)

11. D.M. Bruce, R.N. Hobson, J.W. Farrent and D.G. Hepworth, Compos., Part A Appl. Sci. Manuf., 36, 1486 (2005).

12. B. Wang and M. Sain, Polym. Int., 56, 538 (2007).

13. W.S. Chen, H.P. Yu, Y.X. Liu, P. Chen, M.X. Zhang and Y.F. Hai, Carbohydr. Polym., 83, 1804 (2011).

14. C.K.S. Pillai, W. Paul and C.P. Sharma, Prog. Polym. Sci., 34, 641 (2009).

15. G. Crini and P.-M. Badot, Prog. Polym. Sci., 33, 399 (2008).

16. B. Krajewska, Enzyme Microb. Technol., 35, 126 (2004).

17. M.N.V. Ravi Kumar, React. Funct. Polym., 46, 1 (2000).

18. S. Senel and S.J. McClure, Adv. Drug Deliv. Rev., 56, 1467 (2004).

19. F. Shahidi, J.K.V. Arachchi and Y.-J. Jeon, Trends Food Sci. Technol., 10, 37 (1999).

20. W. Li, J. Yue and S. Liu, Ultrason. Sonochem., 19, 479 (2012).

21. J.M. Lagaro'n and A. Fendler, Film Sheeting, 25, 47 (2009).

22. Y.S. Lu, L.H. Weng and X.D. Cao, Carbohydr. Polym., 63, 198 (2006).

23. A. Khan, R.A. Khan, S. Salmieri, C. Le Tien, B. Riedl, J. Bouchard, G. Chauve, V. Tan, M.R. Kamal and M. Lacroix, Carbohydr. Polym., 90, 1601 (2012).

24. S. Kanagaraj, F.R. Varanda, T.V. Zhil'tsova, M.S.A. Oliveira and J.A.O. Simões, Compos. Sci. Technol., 67, 3071 (2007). 